\title{
Retraction Note: \\ RETRACTED CHAPTER: A Chiral Generic Strategy \\ for Enantioseparation of Acidic and Basic Drugs \\ using Short End Injection Capillary Electrophoresis: \\ Application to Design of Experiment.
}

\author{
Hassan Y. Aboul-Enein and Ahmed M. Abdel-Megied
}

Retraction Note to:

Chapter 9 in: Terry M. Philips (ed.), Clinical Applications of Capillary Electrophoresis: Methods and Protocols, Methods in Molecular Biology, vol. 1972.

https://doi.org/10.1007/978-4939-9213-3_9

The editor has retracted this chapter [1] because it shows significant overlap with a previously published article by Abdel-Megied, Hanafi and Aboul-Enein [2] without proper citation. This chapter is therefore redundant.

Hassan Y. Aboul-Enein does not agree to this retraction/ Ahmed M. Abdel-Megied has not responded to any correspondence from the editor about this retraction.

\section{References}

[1] Aboul-Enein H.Y., Abdel-Megied A.M. (2019) A Chiral Generic Strategy for Enantioseparation of Acidic and Basic Drugs Using Short End Injection Capillary Electrophoresis: Application to Design of Experiment. In: Phillips T. (eds) Clinical Applications of Capillary Electrophoresis. Methods in Molecular Biology, vol 1972. Humana Press, New York, NY

[2] Abdel-Megied, AM, Hanafi, RS, Aboul-Enein, HY. A chiral enantioseparation generic strategy for anti- Alzheimer and antifungal drugs by short end injection capillary electrophoresis using an experimental design approach. Chirality. 2018; 30: 165- 176. https://doi.org/10.1002/chir.22777

The original version of this chapter was retracted. The retraction version of this chapter can be found at: https://doi.org/10.1007/978-4939-9213-3_9 